\title{
Why and how increased plasma ceramides predict future cardiovascular events?
}

\section{Oorni, Katariina}

2020-12

Oorni , K , Jauhiainen , M \& Kovanen , P T 2020 , ' Why and how increased plasma

ceramides predict future cardiovascular events? ' , Atherosclerosis , vol. 314 , pp. 71-73 . https://doi.org/10.1016/j.ath

http://hdl.handle.net/10138/336472

https://doi.org/10.1016/j.atherosclerosis.2020.09.030

cc_by_nc_nd

acceptedVersion

Downloaded from Helda, University of Helsinki institutional repository.

This is an electronic reprint of the original article.

This reprint may differ from the original in pagination and typographic detail.

Please cite the original version. 


\section{Editorial}

\section{Why and how increased plasma ceramides predict future cardiovascular events?}

\section{A R T I C L E I N F O}

\section{Keywords}

Biomarker

Ceramide

Lipoprotein

Sphingomyelinase

Plasma ceramides (CER), a class of sphingolipid molecules, have been identified as markers of future cardiovascular (CV) events and measurement of certain ceramide species and their ratios in plasma samples can predict major acute CV events and CV mortality [1-4]. The data suggest that plasma ceramide levels may predict CV events more accurately than traditional risk biomarkers at least in patients with known atherosclerotic CV disease (CVD). In this issue of Atherosclerosis, $\mathrm{Li}$ et al. [5] have examined in a prospective study the association between various plasma ceramide species and cardiovascular and all-cause mortality in a Chinese cohort of about 1700 CVD patients aged 40-85 years. The authors collected plasma samples, analyzed the concentrations of various ceramide species with HPLC-mass spectrometry method, and followed the patients for an average of 9.3 years. In accordance with data from earlier studies performed largely in Caucasian cohorts, high concentrations of ceramides containing fatty acids 16:0, 18:0, and 24:1 (CER16:0, CER18:0, and CER24:1) and low concentration of CER24:0 were associated with increased cardiovascular mortality. Based on the ratios of CER(16:0/24:0), CER(18:0/24:0), and CER(24:1/24:0), ceramide risk scores have been formulated and found to associate with an increased incidence of future cardiovascular events in earlier studies [1-4], and now also in the study by Li et al. [5].

Ceramides are constituents of cell membranes, act as signal molecules and regulate cellular functions related to cell differentiation, stress, and death, and the related systemic inflammation [6]. The pathophysiological effects of ceramides may include development of insulin resistance and diabetes, enhancement of triglyceride synthesis, and stimulation of cellular apoptosis, e.g. in pancreatic beta cells and cardiomyocytes [7]. In the circulation, ceramides are mostly carried in lipoproteins, where they are situated in the surface monolayer together with apolipoproteins, unesterified cholesterol, and phospholipids. The major site of ceramide synthesis is the liver, where microsomal triglyceride transfer protein (MTP) participates in their targeting to the apolipoprotein B-containing lipoprotein particles and thus their secretion (Fig. 1). Therefore, silencing of the function of the MTP gene in the liver of mice efficiently reduces ceramide plasma levels [8].

Ceramides are minor constituents of the circulating lipoproteins, but their amounts may increase once the lipoproteins have been retained in atherosclerotic lesions, where ceramides are found selectively in aggregated lipoproteins, particularly in aggregated LDL particles [9]. The increased amounts of ceramides are likely a consequence of the activity of arterial extracellular sphingomyelinase, which cleaves sphingomyelins on the surface of the lipoproteins and so generates phosphorylcholines and ceramides [10]. Accumulation of ceramides in lipoprotein particles renders the particles unstable, induces conformational changes in apoB-100, and promotes the formation of very large LDL aggregates [11]. This process is further enhanced if the circulating LDL particles contain high proportions of ceramides and other sphingolipids [12]. In vitro studies have demonstrated that aggregated LDL particles have an increased binding to arterial proteoglycans and are taken up by macrophages with ensuing foam cell formation and inflammasome activation (Fig. 1) [12-14]. Further, cellular ceramides in human coronary atherosclerotic plaques have been located particularly to macrophages located near the necrotic core typical of unstable plaques with a thin fibrous cap [15]. Moreover, uptake of ceramide-containing LDL aggregates induces secretion of matrix metalloproteinase 7 [12], an enzyme linked with plaque rupture [16]. Taking these findings into account, it is understandable that increased levels of plasma ceramides are associated with plaque rupture rather than plaque erosion, i.e., endothelial denudation [17].

Ceramides in extrahepatic tissues may also derive from local synthesis (Fig. 1). In a recent publication, ceramide production by vascular endothelial cells was linked to preserving endothelial functionality and control of blood pressure [18]. The findings in the above-cited work also suggest that a large proportion of the circulating ceramides are produced by endothelial cells. Endothelial cells may directly efflux ceramide to circulating HDL particles, and the phospholipid and cholesteryl ester

DOI of original article: https://doi.org/10.1016/j.atherosclerosis.2020.09.004. 


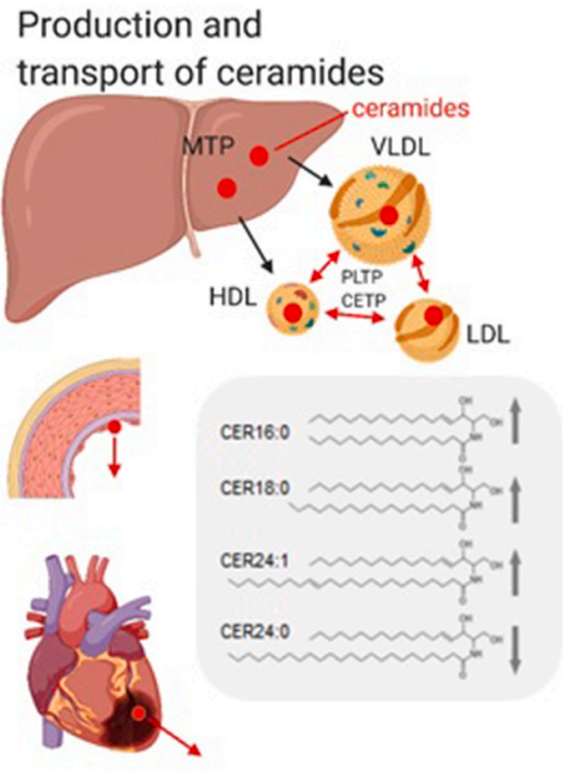

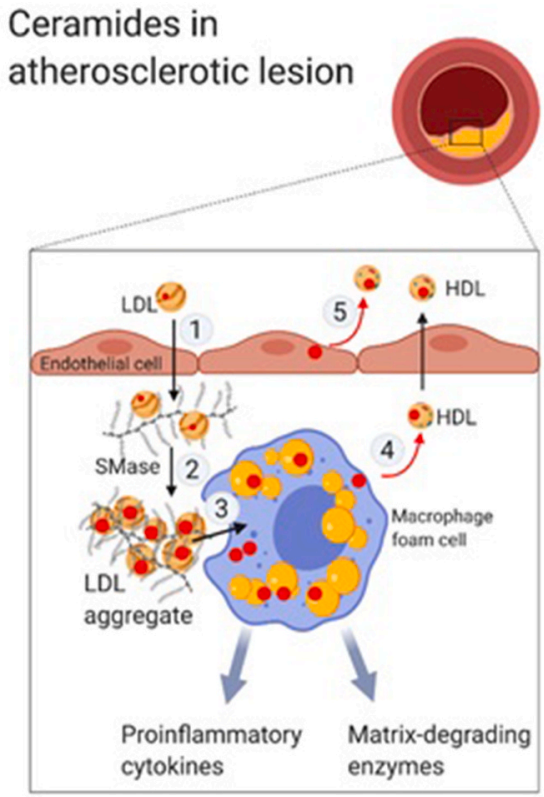

Fig. 1. Production of ceramides, their transport in the circulation, and their effects in an atherosclerotic lesion.

The liver is the main site of ceramide production and the lipoproteins are the main carriers of ceramides in the circulation. In the liver, microsomal triglyceride transfer protein (MTP) plays an important role in ceramide transport to VLDL particles and their secretion into the circulation. Plasma phospholipid and cholesteryl ester transfer proteins (PLTP and CETP) may transfer ceramides between lipoprotein classes. In addition to the liver, also endothelial cells and (ischemic) myocardial tissue have been suggested to contribute to circulating ceramide pool. High concentrations of ceramide (CER) 16:0, CER18:0, and CER24:1 and low concentration of CER24:0 associate with increased cardiovascular disease risk. Ceramides accumulate in atherosclerotic lesions by multiple mechanisms: (1) LDL carries ceramides to the arterial wall, and (2) the action of sphingomyelinase (SMase) increases the local amount of ceramides and also induces the formation of LDL aggregates. (3) Macrophages take up ceramide-containing LDL aggregates, are then converted to foam cells, which show increased contents of ceramides, and increased production and secretion of cytokines and matrixdegrading enzymes.. Efflux of cellular ceramides to HDL particles can take place from (4) foam cells and from (5) endothelial cells. In the figure, red dots depict ceramides and red arrows indicate the fluxes of ceramide molecules and black arrows the transport of ceramides in lipoprotein particles. transfer proteins may then further participate in exchange of ceramides between the circulating lipoproteins. Another source of circulating ceramides has been suggested to be an ischemic myocardial tissue, which thereby may contribute to an increase in the plasma levels of ceramides after an acute myocardial infarction [4]. This suggestion is supported by observations in rat models with myocardial ischemia showing that together with increased myocardial ceramide levels also the plasma levels of ceramides are increased $[4,19,20]$.

In humans, ceramide synthesis is increased in various metabolic disorders, such as in metabolic syndrome and type 2 diabetes, and, moreover, ceramide synthesis is also influenced by the diet [7]. Thus, overeating saturated fat increases the ceramide levels both in the circulation and in the liver, and also promotes non-alcoholic fatty liver disease [21-23]. Interestingly, a Mediterranean dietary intervention may temper potential detrimental effects of elevated plasma ceramide concentrations on CVD [24]. Alterations in ceramide metabolism are also linked to insulin resistance, metabolism of the adipose tissue, and systemic inflammation. Determination of the exact mechanisms behind the altered ceramide metabolism may lead to the development of novel treatment options for the metabolic diseases involved.

The present prospective study by Li et al. [5] confirms the benefit and added value of analyzing certain plasma ceramide species when evaluating the risk of CVD and all-cause mortality. These ceramides have been suggested to outperform conventional CVD risk biomarkers at least in patients with known CVD, and some clinics have already launched measurement of serum ceramides as a means of identifying at-risk patients. As many lines of evidence point to an active role for ceramides in the development and progression of atherosclerosis and its complications, ceramides may also be an attractive treatment target in atherosclerotic cardiovascular disease.

\section{Declaration of competing interest}

The authors declare that they have no known competing financial interests or personal relationships that could have appeared to influence the work reported in this paper.

\section{Acknowledgements}

Wihuri Research Institute is maintained by the Jenny and Antti Wihuri Foundation. The research is also supported by grants from the Academy of Finland (\#315568 and \#332564) and the Finnish Foundation for Cardiovascular Research to KÖ and from Jane and Aatos Erkko Foundation to MJ.

\section{References}

[1] R. Laaksonen, K. Ekroos, M. Sysi-Aho, et al., Plasma ceramides predict cardiovascular death in patients with stable coronary artery disease and acute coronary syndromes beyond LDL-cholesterol, Eur. Heart J. 37 (2016) 1967-1976.

[2] L.R. Peterson, V. Xanthakis, M.S. Duncan, et al., Ceramide remodeling and risk of cardiovascular events and mortality, J Am Heart Assoc 7 (2018).

[3] A.S. Havulinna, M. Sysi-Aho, M. Hilvo, et al., Circulating ceramides predict cardiovascular outcomes in the population-based FINRISK 2002 cohort, Arterioscler. Thromb. Vasc. Biol. 36 (2016) 2424-2430.

[4] L.P. de Carvalho, S.H. Tan, G.S. Ow, et al., Plasma ceramides as prognostic biomarkers and their arterial and myocardial tissue correlates in acute myocardial infarction, JACC Basic Transl Sci 3 (2018) 163-175.

[5] Q. Li, X. Wang, J. Pang, et al., Associations between Plasma Ceramides and Mortality in Patients with Coronary Artery Disease, 2020. Atherosclerosis.

[6] M.N. Nikolova-Karakashian, K.A. Rozenova, Ceramide in stress response, Adv. Exp. Med. Biol. 688 (2010) 86-108.

[7] S.A. Summers, B. Chaurasia, W.L. Holland, Metabolic messengers: ceramides, Nat Metab 1 (2019) 1051-1058.

[8] J. Iqbal, M.T. Walsh, S.M. Hammad, et al., Microsomal triglyceride transfer protein transfers and determines plasma concentrations of ceramide and sphingomyelin but not glycosylceramide, J. Biol. Chem. 290 (2015) 25863-25875.

[9] S.L. Schissel, J. Tweedie-Hardman, J.H. Rapp, et al., Rabbit aorta and human atherosclerotic lesions hydrolyze the sphingomyelin of retained low-density lipoprotein. Proposed role for arterial-wall sphingomyelinase in subendothelial retention and aggregation of atherogenic lipoproteins, J. Clin. Invest. 98 (1996) $1455-1464$.

[10] S.L. Schissel, E.H. Schuchman, K.J. Williams, et al., Zn2+-stimulated sphingomyelinase is secreted by many cell types and is a product of the acid sphingomyelinase gene, J. Biol. Chem. 271 (1996) 18431-18436. 
[11] M. Sneck, S.D. Nguyen, T. Pihlajamaa, et al., Conformational changes of apoB-100 in SMase-modified LDL mediate formation of large aggregates at acidic pH, JLR ( J. Lipid Res.) 53 (2012) 1832-1839.

[12] M. Ruuth, S.D. Nguyen, T. Vihervaara, et al., Susceptibility of low-density lipoprotein particles to aggregate depends on particle lipidome, is modifiable, and associates with future cardiovascular deaths, Eur. Heart J. 39 (2018) 2562-2573.

[13] K. Öörni, M.O. Pentikäinen, M. Ala-Korpela, et al., Aggregation, fusion, and vesicle formation of modified low density lipoprotein particles: molecular mechanisms and effects on matrix interactions, J. Lipid Res. 41 (2000) 1703-1714.

[14] S. Lehti, D. Nguyen, I. Belevich, et al., Extracellular lipid accumulates in human carotid arteries as distinct three-dimensional structures with proinflammatory properties, Am. J. Pathol. 188 (2018) 525-538.

[15] Y. Uchida, Y. Uchida, T. Kobayashi, et al., Detection of ceramide, a risk factor for coronary artery disease, in human coronary plaques by fluorescent angioscopy, Circ. J. 81 (2017) 1886-1893.

[16] A. Abbas, P. Aukrust, D. Russell, et al., Matrix metalloproteinase 7 is associated with symptomatic lesions and adverse events in patients with carotid atherosclerosis, Plos One 9 (2014), e84935.

[17] W. Pan, M. Sun, J. Wu, et al., Relationship between elevated plasma ceramides and plaque rupture in patients with ST-segment elevation myocardial infarction, Atherosclerosis 302 (2020) 8-14.

[18] A. Cantalupo, L. Sasset, A. Gargiulo, et al., Endothelial sphingolipid de novo synthesis controls blood pressure by regulating signal transduction and NO via ceramide, Hypertension 75 (2020) 1279-1288.

[19] D.X. Zhang, R.M. Fryer, A.K. Hsu, et al., Production and metabolism of ceramide in normal and ischemic-reperfused myocardium of rats, Basic Res. Cardiol. 96 (2001) 267-274.

[20] A. Beresewicz, A. Dobrzyn, J. Gorski, Accumulation of specific ceramides in ischemic/reperfused rat heart; effect of ischemic preconditioning, J. Physiol. Pharmacol. 53 (2002) 371-382.

[21] P.K. Luukkonen, S. Sadevirta, Y. Zhou, et al., Saturated fat is more metabolically harmful for the human liver than unsaturated fat or simple sugars, Diabetes Care 41 (2018) 1732-1739.
[22] P.K. Luukkonen, Y. Zhou, S. Sadevirta, et al., Hepatic ceramides dissociate steatosis and insulin resistance in patients with non-alcoholic fatty liver disease, J. Hepatol. 64 (2016) 1167-1175.

[23] F. Rosqvist, J. Kullberg, M. Stahlman, et al., Overeating saturated fat promotes fatty liver and ceramides compared with polyunsaturated fat: a randomized trial, J. Clin. Endocrinol. Metab. 104 (2019) 6207-6219.

[24] D.D. Wang, E. Toledo, A. Hruby, et al., Plasma ceramides, Mediterranean diet, and incident cardiovascular disease in the PREDIMED trial (Prevention con Dieta Mediterranea), Circulation 135 (2017) 2028-2040.

Katariina Ö̈rni

Atherosclerosis Research Laboratory, Wihuri Research Institute, Helsinki,

Finland

Molecular and Integrative Biosciences Research Programme, Faculty of Biological and Environmental Sciences, University of Helsinki, Helsinki,

Finland

Matti Jauhiainen

Minerva Foundation Institute for Medical Research, Helsinki, Finland

Petri T. Kovanen

Atherosclerosis Research Laboratory, Wihuri Research Institute, Helsinki,

Finland

* Corresponding author. Atherosclerosis Research Laboratory Wihuri Research Institute Biomedicum I Haartmaninkatu, 8 00290, Helsinki,

Finland.

E-mail address: kati.oorni@wri.fi (K. Öörni). 